\title{
Protocolo del estudio: Demanda y práctica farmacéutica en afección bucofaríngea en España. Estudio ACTUA
}

\author{
Antonio Hernández Rex', Pilar García-Delgado², Ana Ocaña Arenas², Victoria García-Cárdenas², \\ Elena Labrador Barba ${ }^{3}, \mathrm{M}^{\mathrm{a}}$ Luisa Orera Peña ${ }^{3}$, Fernando Martínez-Martínez ${ }^{2}$ \\ 1. Farmacéutico comunitario en Murcia. 2. Grupo de Investigación en Atención Farmacéutica de la Universidad de Granada. 3. Departamento Médico \\ de Abbott Laboratories S.A.
}

\section{PALABRAS CLAVE}

afección bucofaríngea, atención farmacéutica, dispensación, indicación farmacéutica, farmacia comunitaria

\section{KEYWORDS}

oropharyngeal disease, pharmacy practice, dispensing, counseling, community pharmacy

\section{RESUMEN}

Introducción y justificación: La afección bucofaríngea, y más concretamente el dolor de garganta, por su prevalencia y relación con el uso inadecuado de medicamentos tiene una alta importancia en salud, el farmacéutico contribuye a que el usuario alcance una automedicación adecuada a través de los servicios de atención farmacéutica. Sin embargo, se conoce poco en el ámbito de la farmacia comunitaria sobre los usuarios que demandan esta atención, así como las consultas e intervenciones de los farmacéuticos.

Aplicabilidad de los resultados: Conocer las características de la demanda y la práctica farmacéutica en afección bucofaríngea permitirá establecer estrategias sanitarias destinadas a optimizar la asistencia sanitaria.

Objetivos: Caracterizar la práctica farmacéutica en afección bucofaringea realizada en farmacias comunitarias españolas.

Material y métodos: Estudio observacional descriptivo transversal. En farmacias comunitarias voluntarias del territorio español. La población de estudio serán los usuarios que acudan a las farmacias por una afección bucofaríngea. La duración del trabajo de campo será de tres meses. Las variables contempladas en el estudio serán aquellas que caracterizan al usuario, a la consulta realizada, y a la intervención del farmacéutico. Se realizará un análisis estadístico descriptivo de los datos (univariante y multivariante por la técnica de correspondencias múltiples). Se garantizará la confidencialidad y el consentimiento informado de los participantes.

Study protocol: demand and pharmaceutical practice in buccopharyngeal disorders in Spain. The ACTUA study

\section{ABSTRACT}

Introduction and rationale: Due to their prevalence and association to inadequate medications use, buccopharyngeal disorders - and specifically sore throat - are an important health concern. In this regard, the pharmacist contributes to ensure adequate patient self-medication through pharmaceutical care services. However, in the community pharmacy setting, little is known of the users who demand such care and of pharmacist consultations and interventions.

Applicability of the results: Knowing the characteristics of the demands and pharmaceutical practices in buccopharyngeal disorders will allow the definition of healthcare strategies designed to optimize patient care.

Objectives: To characterize pharmaceutical practice in buccopharyngeal disorders in Spanish community pharmacies.

Materials and methods: A cross-sectional, descriptive observational study will be carried out involving the voluntary participation of community pharmacies in Spain. The study population will consist of those users visiting pharmacies due to buccopharyngeal disorders. The field study will last three months. The study variables will comprise the characteristics of the user, the consultation made, and pharmacist intervention. A descriptive statistical study will be made involving uni- and multivariate analysis based on the multiple correspondence technique. Confidentiality of the participants will be observed, and informed consent will be obtained.
Recibido: $10 / 4 / 2014$

Aceptado: $17 / 7 / 2014$

Disponible online: 1-12-2014
Financiación: El estudio será financiado por Abbott Laboratories S.A.

Conflicto de intereses: Los autores declaran no existir conflicto de intereses en relación con el contenido del presente trabajo. Cite este artículo como: Hernández A, Garcia-Delgado P, Ocaña A, Garcia-Cárdenas V, Labrador E, Orera ML, Martinez-Martinez F. Protocolo del estudio: Demanda y práctica farmacéutica en afección bucofaringea en España. Estudio ACTUA. Farmacéuticos Comunitarios. 2014 Dec 01; 6(4):21-25. doi:10.5672/FC.2173-9218.(2014/Vol6).004.04

Autor para correspondencia: Pilar Garcia Delgado (pgarciadelgado@yahoo.es).

ISSN 1885-8619 OSEFAC (Sociedad Española de Farmacia Familiar y Comunitaria). Todos los derechos reservados. 


\section{Introducción}

Las afecciones bucofaringeas engloban todas aquellas dolencias localizadas en la boca, la faringe o la laringe. Se manifiestan generalmente como "dolor de garganta” y se deben, mayoritariamente, a una infección aguda del tracto respiratorio superior que daña a la mucosa respiratoria. La infección puede afectar a cualquier parte de la mucosa de forma aislada (faringitis o amigdalitis), o verse todas las áreas afectadas (simultáneamente o en momentos diferentes). Estas afecciones suelen estar asociadas con dolor de cabeza, fiebre, malestar general, sintomas que aunque frecuentemente resultan ser incapacitantes para el paciente, y que son considerados frecuentemente como sintomas menores (1). Los síntomas de dolor de garganta desaparecen en 3 días sin tratamiento en aproximadamente el $40 \%$ de las personas y la fiebre en alrededor del 85\% (2). En una semana, el 85\% de las personas no presenta síntomas. Por este motivo el dolor de garganta se considera un sintoma menor susceptible de autocuidados. Sin embargo, en ocasiones puede agravarse originando complicaciones supurativas como la otitis media aguda, sinusitis aguda, y el absceso periamigdalino $\mathrm{y} / \mathrm{o}$ complicaciones no supurativas como la fiebre reumática aguda y la glomerulonefritis aguda.

La importancia en salud de este problema se deriva de una alta prevalencia. Un estudio en Australia determinó que en un año, aproximadamente el 10\% de la población consulta a los servicios de atención primaria por una infección del tracto respiratorio superior entre las que predominan, cuando solo se menciona un síntoma, las de dolor de garganta (3). En Escocia se ha encontrado que el $31 \%$ de los adultos estudiados reportó un severo dolor de garganta en el año anterior, para los cuales el 38\% de estas personas visitó a un médico (4).

En España, el dolor de garganta supone 4 millones de consultas al año. La tercera parte de las consultas a atención primaria se deben a procesos infecciosos y más de la mitad de éstas se deben a infecciones de las vías respiratorias altas (5). La Encuesta Nacional de Salud del año 2006 (6) identificaba a los analgésicos, los medicamentos para el resfriado, gripe $y$ fiebre o las asociaciones para síntomas de procesos gripales o ca- tarrales, como los medicamentos más utilizados para automedicación por los españoles. La prevalencia de automedicación en mayores de 16 años, en España, es de un 12,7\% aunque los autores calificaban como "automedicación indeseable" o no apropiada a un 2,5\% de la muestra (7).

Un ejemplo de la automedicación inadecuada corresponde al uso de antibióticos para el dolor de garganta sin que medie prescripción. De hecho, los antibióticos sistémicos son uno de los fármacos más demandado sin receta médica en las farmacias (8). Grigoryan et al (9) determinó la prevalencia de automedicación con antibióticos sistémicos así como la intención de automedicarse en Europa. La razón más común que los pacientes esgrimieron para la automedicación fue la creencia de que el tratamiento con antibióticos es adecuado para ciertos síntomas menores, como el dolor de garganta (10).

La inadecuada automedicación constituye un problema de salud pública mundial.

El proceso de gestión de síntomas menores supone el uso de tres tipos de recursos en atención primaria: el médico general, el farmacéutico comunitario y los autocuidados (11).

El profesional farmacéutico es visto por los pacientes como un consejero en el cuidado de su salud con un papel complementario al médico, proporcionando información que el médico no da, o resolviendo dudas en cuanto a los medicamentos (12), asumiendo su papel de experto en- medicamentos (13). Asimismo, se ha expuesto que los pacientes prefieren tratar los síntomas menores o banales en las farmacias (14), escogiendo al médico de atención primaria cuando entienden que el problema de salud que padecen no es apropiado que lo trate un farmacéutico (15).

Por lo tanto, se puede afirmar que el farmacéutico comunitario es frecuentemente el primer punto de contacto del paciente con el sistema sanitario en el tratamiento de síntomas menores. Su accesibilidad y disponibilidad lo convierten en el asesor ideal para este tipo de eventos, donde representan un facilitador ya sea confirmando la elección de la automedicación, seleccionando una terapia o al actuar como filtro derivando al médico general ante situaciones más graves (16).

\section{Justificación}

La función del farmacéutico comunitario dentro del sistema sanitario ha variado en los últimos años hacia un modelo de práctica profesional enmarcado dentro del concepto de la atención farmacéutica, definida como "la participación activa del farmacéutico en la mejora de la calidad de vida del paciente, mediante la dispensación, indicación farmacéutica y seguimiento farmacoterapéutico" (17). En España se han consensuado tres servicios esenciales, englobados dentro de este concepto, relacionados con el modelo actual de ejercicio profesional del farmacéutico y orientados a las necesidades del paciente (17) (tabla 1).

Tabla 1 Servicios profesionales englobados en el marco de la atención farmacéutica en España

Servicio de dispensación

Es el servicio profesional del farmacéutico encaminado a garantizar, tras una evaluación individual, que los pacientes reciban y utilicen los medicamentos de forma adecuada a sus necesidades clínicas, en las dosis precisas según sus requerimientos individuales, durante el periodo de tiempo adecuado, con la información para su correcto uso y de acuerdo con la normativa vigente.

Servicio de indicación farmacéutica

Es el servicio profesional prestado ante la demanda de un paciente o usuario que llega a la farmacia sin saber qué medicamento debe adquirir y solicita al farmacéutico el remedio más adecuado para un problema de salud concreto.

\section{Seguimiento farmacoterapéutico}

Es el servicio profesional que tiene como objetivo la detección de problemas relacionados con medicamentos (PRM), para la prevención y resolución de resultados negativos asociados a la medicación (RNM). Este servicio implica un compromiso y debe proveerse de forma continuada, sistematizada y documentada, en colaboración con el propio paciente y con los demás profesionales del sistema de salud, con el fin de alcanzar resultados concretos que mejoren la calidad de vida del paciente. 
Por ello, ante la presencia de una afección bucofaríngea, e independientemente de la opción elegida por el paciente para manejarla, el farmacéutico debe seleccionar cualquiera de estos tres servicios asistenciales, contribuyendo e interviniendo en la cadena asistencial del mismo, bien procediendo a la entrega de un medicamento o bien asesorando al paciente sobre su situación. A pesar de la elevada prevalencia de las afecciones bucofaríngeas y su relación con el uso inadecuado de la medicación, en nuestro conocimiento, no existe ningún estudio que haya analizado la práctica farmacéutica en este tipo de afecciones.

\section{Aplicabilidad de los resultados}

Los resultados obtenidos permitirán evaluar la demanda y solicitudes planteadas por los pacientes con afección bucofaríngea en la farmacia comunitaria. Detectando con ello las necesidades asistenciales en esta patología. Del mismo modo, se podrá determinar las intervenciones realizadas por los farmacéuticos comunitarios en pacientes con afección bucofaríngea, contribuyendo a diseñar estrategias de política sanitaria para solventar las posibles deficiencias encontradas y optimizar la gestión sanitaria farmacéutica de este síntoma menor.

\section{Objetivos}

\section{Objetivo general}

- Caracterizar la práctica farmacéutica en afección bucofaríngea prestada en farmacias comunitarias españolas en cuanto a los usuarios demandantes, las solicitudes efectuadas y las intervenciones farmacéuticas recibidas.

Para lo que se establecen los siguientes objetivos específicos:

- Describir las características sociodemográficas de los usuarios con afección bucofaríngea que acuden a las farmacias comunitarias españolas para su tratamiento.

- Analizar las características de la demanda de los usuarios, el servicio (dispensación y/o indicación) y producto solicitado, origen de la solicitud y síntoma consultado.

- Describir las intervenciones de los farmacéuticos participantes en cuanto a los medicamentos seleccionados y la derivación a otros profesionales sanitarios.

\section{Material y método}

Diseños del estudio

Estudio observacional descriptivo transversal multicéntrico.

\section{Ámbito de estudio}

Farmacias comunitarias voluntarias del territorio español seleccionadas por la red comercial del laboratorio Abbott.

Población de estudio

Usuarios que acudan a la farmacia comunitaria participante por una afección bucofaríngea.

\section{Criterios de inclusión}

- Usuarios que acudan a las farmacias comunitarias incluidas en el estudio realizando una consulta relacionada con la afección bucofaríngea o demandando algún tratamiento para la afección bucofaríngea (con o sin receta médica).

\section{Criterios de exclusión}

- Pacientes con dificultades de comunicación, psicológicas o lingüísticas.

- Usuarios menores de 18 años.

- Pacientes que actualmente estén participando en algún ensayo clínico.

- Pacientes con dolor de garganta asociado a enfermedad sistémica o grave.

- Aquellos pacientes que hayan participado anteriormente en este estudio no serán excluidos pero se anotará dicha circunstancia.

\section{Tamaño muestral}

Debido a los objetivos del estudio, no es necesario el cálculo del tamaño muestral. Se realizará un muestreo intencional en el que se incluirán, del total de farmacias comunitarias del territorio español, 1.000 farmacias que acepten voluntariamente participar en el estudio en cada una de las cuales se seleccionarán los pacientes con afección bucofaríngea que cumplan los criterios de inclusión y que acepten participar hasta completar un total de cinco usuarios.

\section{Duración del estudio}

El trabajo de campo se realizará en un plazo de tres meses dado que la incidencia de la afección bucofaríngea está sujeta a la estacionalidad, el periodo de recogida de la información será en los meses de noviembre a marzo.

\section{Variables}

Las variables contempladas en el estudio se clasifican en: relacionadas con el farmacéutico, relacionadas con la demanda y relacionadas con el usuario.

\section{Relacionadas con el farmacéutico}

Edad. Entendida como años cumplidos por el farmacéutico en el momento de inicio del trabajo de campo. Variable cuantitativa discreta.

Sexo. Sexo del farmacéutico. Variable dicotómica cuyas categorías son: hombre/mujer

Años de experiencia profesional en farmacia comunitaria. Entendido como años trabajados como farmacéutico en el ámbito de la farmacia comunitaria. Variable cuantitativa discreta.

Formación continuada acreditada. Entendida como actualización de los conocimientos en atención farmacéutica en los últimos 5 años con título que lo acredite. Variable categórica dicotómica cuyas categorías son: Sí/No.

\section{Relacionadas con la demanda} Servicio de atención farmacéutica solicitado. Variable categórica:

- Dispensación, entendida como la solicitud de un medicamento o producto (por su nombre comercial) para la afección bucofaríngea, ya sea con receta médica o sin ella.

- Indicación farmacéutica, entendida como la solicitud por parte del usuario de la selección de un tratamiento por parte del farmacéutico para la afección bucofaríngea.

Medicamento y/o producto solicitado por el usuario. Entendido como medicamento o producto sanitario del que el usuario (en su caso) solicita la dispensación. Variable categórica. Se categorizará por grupos terapéuticos según el Sistema de Clasificación Anatómica, Terapéutica y Química (ATC). Se recogerá el código nacional de los medicamentos mediante item abierto. 
Origen de la recomendación. Entendido como persona de la que parte la recomendación del medicamento o producto del que el usuario solicita la dispensación. Variable categórica: Médico/ Farmacéutico/ Enfermero/ Otros profesionales sanitarios/ Automedicación/ Familiares o amigos.

Razón de consulta. Problema de salud por el que el usuario solicita la atención farmacéutica. Se recoge mediante item abierto. Posteriormente se categorizará según la clasificación CIAP-2.

Síntoma bucofaríngeo. Entendido como problema de salud banal, por el que el usuario demanda el servicio de indicación farmacéutica. Variable policotómica, categorizado en: Dolor de garganta/ Aftas/ Afonía/ Otros.

Duración del síntoma. Percepción del paciente sobre los días de duración del problema de salud en el momento que consulta al farmacéutico. Variable continúa.

Intervención farmacéutica. Actuación profesional del farmacéutico ante la demanda del usuario. Variable categórica (múltiple respuesta): Entrega del medicamento solicitado/ Selección de tratamiento farmacológico/ Selección de tratamiento no farmacológico/ Derivación a otro profesional sanitario

Medicamento o producto sanitario que indica el farmacéutico. Entendido como medicamento o producto sanitario que en su caso seleccionó el farmacéutico para tratar el problema de salud del paciente. Variable categórica. Se categorizó por grupos terapéuticos según el sistema (ATC). Se recogerá el código nacional de los medicamentos seleccionados por los farmacéuticos mediante ítem abierto

Motivo de derivación. Razón por la que el farmacéutico decide derivar al paciente a otro profesional. Variable categórica. Se recogerá mediante ítem abierto. Posteriormente, se establecerán las categorías para el análisis de la información.

3. Relacionadas con el usuario demandante.

Persona que realiza la consulta. Variable dicotómica:

- Paciente. Se entiende como paciente aquella persona que consulta por un problema de salud que padece él mismo.

- Cuidador. Se entiende por cuidador aquella persona que se responsabi- liza y asume el cuidado de un paciente, sea familiar o no.

- Tercera persona. Se entiende como tercera persona aquella que no es ni paciente, ni cuidador.

Edad. Años cumplidos por el usuario en el momento de la consulta en la farmacia. Variable discreta.

Sexo del usuario. Variable dicotómica. Categorias: Mujer/Hombre.

Nivel de educación. Entendido como estudios finalizados por el usuario. Variable ordinal. Categorizado en:

- Sin estudios.

- Estudios primarios (graduado escolar, primaria).

- Estudios secundarios (secundaria, formación profesional).

- Estudios universitarios.

Número de medicamentos. Entendido como el número de medicamentos que toma el paciente en el momento de la consulta. Variable cuantitativa discreta.

Profesión. Entendida como profesión a la que, en el momento de la entrevista, se dedica el paciente. Variable categórica (clasificación del Centro de Investigaciones Sociológicas).

\section{Fuentes de información}

Dos formularios diseñados ad hoc en el que se recogerán las distintas variables del estudio:

- Formulario de datos del farmacéutico (un cuestionario por farmacéutico colaborador).

- Formulario de datos del paciente (uno por paciente), datos de la consulta efectuada y de la intervención del farmacéutico. Lo cumplimentará el farmacéutico colaborador tras su intervención con el paciente.

\section{Interacción farmacéutico- paciente}

El farmacéutico colaborador ofrecerá la participación en el estudio a todos aquellos pacientes que cumplan los criterios de inclusión, y tras una breve explicación del proyecto de investigación le pedirá su colaboración para la participación en el mismo, firmando finalmente el consentimiento informado.

Estos pacientes recibirán la atención habitual por parte de los farmacéuticos colaboradores, que cumplimentarán las variables de estudio recogidas en los formularios. Una vez finalizado el trabajo de campo los formularios se enviarán al equipo investigador para su evaluación y codificación.

\section{Análisis estadístico}

La explotación de la base de datos creada se analizará con el programa estadístico SPSS ${ }^{\circledR}$ 15.0. Se realizará un análisis descriptivo univariante, mostrando la frecuencia de aparición de las distintas variables en la población de estudio, así como de las pérdidas producidas durante el trabajo de campo. Para las variables cualitativas se obtendrán tablas de frecuencias absolutas y relativas, y para las cuantitativas, medidas de tendencia central (media), medidas de dispersión (desviación estándar) y medidas de posición (cuartiles). Se calcularán estimaciones poblacionales así como sus correspondientes errores de muestreo para el cálculo de los intervalos de confianza. Por último se realizará un análisis multivariante por la técnica de correspondencias múltiples, con el objetivo de analizar la posible asociación entre el perfil del farmacéutico y la intervención realizada.

\section{Limitaciones}

Este estudio no pretende ser representativo del número de consultas farmacéuticas realizadas por los usuarios de las farmacias comunitarias españolas, no obstante pretende explorar qué tipo de consultas sobre afección bucofaríngea se realizan, por este motivo las farmacias seleccionadas son voluntarias, priorizando el compromiso de cada farmacéutico en la cumplimentación del estudio

\section{Consideraciones éticas}

La investigación está financiada por Laboratorios Abbott. Sin embargo, la fuente financiadora no influirá en la obtención de los resultados, minimizando el conflicto de intereses.

Este estudio ha sido aprobado por el Comité Ético de Investigación Clínica del Hospital Clinic de Barcelona.

\section{Confidencialidad}

Se garantizará la confidencialidad de la información utilizada en la investigación como aspecto ético clave. 
Por tanto, el objetivo de la protección de datos es doble:

- Garantizar la exclusiva utilización de la información para los objetivos de investigación planteados;

- Garantizar los derechos de los participantes evitando que pudiera vincularse la identificación de la persona con sus datos personales.

\section{Consentimiento}

Se respetará la autonomía de la persona que participa en el estudio y su capacidad de decisión libre e informada, por ello se solicitará el consentimiento informado. Así, el farmacéutico colaborador proporcionará la información suficiente para garantizar la comprensión, por parte del usuario, de los objetivos del estudio, lo que supone la participación en el mismo y todo lo que la persona requiera. Una vez satisfecha la comprensión por parte de la persona, se solicitará su participación voluntaria, expresada en la firma del formulario.

\section{Agradecimientos}

A la coordinación técnica del Grupo SANED. Al patrocinio de Abbott Laboratories S.A.

\section{Referencias bibliográficas}

1. Paudyal V, Hansford D, Cunningham S, Stewart D. Pharmacy assisted patient self care of minor ailments: a chronological review of UK health policy documents and key events 1997-2010. Health Policy. 2011 Aug;101(3):253-9. doi:10.1016/j.healthpol.2011.05.010

2. Del Mar CB, Glasziou PP, Spinks AB. Antibiotics for sore throat. Cochrane Database Syst Rev. 2006(4):CD000023.
3. Benediktsdottir B. Upper airway infections in preschool children--frequency and risk factors. Scand J Prim Health Care. 1993 Sep;11(3):197-201. doi:10.3109/02813439308994830

4. Hannaford PC, Simpson JA, Bisset AF, Davis A, McKerrow W, Mills R. The prevalence of ear, nose and throat problems in the community: results from a national cross-sectional postal survey in Scotland. Fam Pract. 2005 Jun;22(3):227-33. doi:10.1093/fampra/cmi004

5. Llor C. Uso prudente de antibióticos y propuestas de mejora desde la atención primaria. Enferm Infecc Microbiol Clin. Nov;28(Suppl 4):17-22.

6. Encuesta nacional de salud 2006. [Internet]. Madrid: Ministerio de Sanidad y Consumo. 2008. [acceso 8/1/2014]. Disponible en: http://www. msps.es/estadEstudios/estadisticas/ encuestaNacional/encuestaNac2006/ encuestaNacionalSalud2006.pdf

7. Figueiras A, Caamano F, Gestal-Otero JJ. Sociodemographic factors related to self-medication in Spain. Eur J Epidemiol. 2000 Jan;16(1):19-26. doi:10.1023/A:1007608702063

8. Barbero-Gonzalez A, Pastor-Sanchez R, del Arco-Ortiz de Zarate J, Eyaralar-Riera T, Espejo-Guerrero J. Demanda de medicamentos de prescripción sin receta médica. Aten Primaria. 2006 Feb 15;37(2):78-87. doi:10.1157/13084485

9. Grigoryan L, Haaijer-Ruskamp FM, Burgerhof JG, Mechtler R, Deschepper R, Tambic-Andrasevic A, et al. Self-medication with antimicrobial drugs in Europe. Emerg Infect Dis. 2006 Mar;12(3):452-9. doi:10.3201/ eid1203.050992

10. Grigoryan L, Burgerhof JG, Degener JE, Deschepper R, Lundborg CS, Monnet DL, et al. Determinants of self-medication with antibiotics in Europe: the impact of beliefs, country wealth and the healthcare system. J Antimicrob Chemother. 2008
May;61(5):1172-9. doi:10.1093/jac/ dkn054

11. Hassell K, Rogers A, Noyce P. Community pharmacy as a primary health and self-care resource: a framework for understanding pharmacy utilization. Health \& Social Care in the Community. 2000;8(1):40-9. doi:10.1046/ j.1365-2524.2000.00222.x

12. Fundación Josep Laporte. The Future Patient - Resultado del Focus group español [Internet]. 2001. [acceso 8/1/2014]. Disponible en: http://www. fbjoseplaporte.org/2008/publicacions/ index.php?secc $=$ fitxaftid $=18$.día

13. Anderson C, Blenkinsopp A, Armstrong M. Feedback from community pharmacy users on the contribution of community pharmacy to improving the public's health: a systematic review of the peer reviewed and non-peer reviewed literature 1990-2002. Health Expect. 2004 Sep;7(3):191-202. doi:10.1111/j.13697625.2004.00274.x

14. Llanes de Torres R, Aragón Pena A, Sillero Quintana MI, Martín Rios MD. ¿Ir al centro de salud o bajar a la farmacia? Las oficinas de farmacia como recurso de atención primaria. Aten Primaria. 2000 Jun 15;26(1):11-5. doi:10.1016/S0212-6567(00)78598-2

15. Hammond T, Clatworthy J, Horne R. Patients' use of GPs and community pharmacists in minor illness: a cross-sectional questionnaire-based study. Fam Pract. 2004 Abr;21(2):1469. doi:10.1093/fampra/cmh207

16. Campbell A, McGovern EM, Millar H, Bryson SM. SIGN guidelines and the management of sore throat in community pharmacy. International Journal of Pharmacy Practice. 2001;9:15. doi:10.1111/j.2042-7174.2001. tb01075.x.

17. Grupo de Expertos de Foro de Atención Farmacéutica. Documento de Consenso. Madrid: Consejo General de Colegios Oficiales de Farmacéuticos; 2008. 